\title{
Survey about end-of-life decision in the general population
}

\author{
Luz María Guadalupe Pichardo-García ${ }^{1 *}$, María de la Luz Lina Casas-Martínez², \\ Mónica Jaimes-Palomera ${ }^{3}$, Alma Guadalupe Sotelo-Méndez ${ }^{4}$, Ana Paula Sosa-Delgado4, \\ Andrea Quintero-Luna, ${ }^{4}$ Tanit lanie López-Vergara-Anaya ${ }^{4}$ and José Alberto Aguilar-Júarez ${ }^{3}$ \\ ${ }^{1}$ Universidad Panamericana, Faculty of Law, Pan American Institute of Jurisprudence; '2 Universidad Panamericana, Faculty of Health Sciences, \\ Interdisciplinary Center of Bioethics; ${ }^{3}$ Secretaría del Medio Ambiente de la Ciudad de México, Sub-directorate of Analysis; ${ }^{4}$ Universidad Panamericana, \\ Faculty of Health Sciences, Nursing School. Ciudad de México, Mexico
}

\begin{abstract}
Introduction: Concepts related to end-of-life decisions, such as euthanasia, palliative care, advance directives and therapeutic obstinacy, are poorly understood by the general population, which, when facing a terminal situation, is not prepared to choose the best option. Objective: Pilot study $(n=544)$ to find out what the open population understands about terms used in end-of-life situations in four cities of the Mexican Republic. Method: Survey via Internet with 18 questions about different terms. It was a descriptive, cross-sectional study. Statistical analysis was carried out. Results: People older than 18 years who were not engaged in health-related professional activities were selected. Conclusions: Most terms related to end-of-life decisions were found not to be interesting to or understood by a part of the population. The least recognized term was therapeutic obstinacy (62.8\%), and the most widely known, palliative care (91\%); there was confusion between the terms euthanasia and assisted suicide (47.8\%). Age and education level had more influence in the results, than other demographic variables.
\end{abstract}

KEY WORDS: End-of-life. Advance directive. Euthanasia. Palliative care. Therapeutic obstinacy. General population.

\section{Introduction}

To adequately exercise our autonomy, one of the most important aspects is full understanding of that which we are being questioned about. When the options contain specific terminology of some field of knowledge, it occurs that those who are foreign to it, poorly understand its meaning, which makes for the faculty of free and informed decision to become questionable. As Cano Valle points out: ${ }^{1}$

Even if we establish a conceptual statement about freedom, in the reality of Mexico, and of Latin America in general, the levels of social exclusion and inequality are closely related to the varying degrees of autonomy. Low educational level, poverty and non-accessibility to health services constitute a perverse triad of social isolation we have to fight against, since it cannot and should not be a perpetual state that defines developing countries. It is urgent to alleviate certain needs and correct some fragilities as soon as possible; the sooner, the better.

For this reason, the purpose of this work was to investigate the understanding of terms and postures related to end of life in the general population in four states of the Mexican Republic.

In the last five years, in different Spanish-speaking countries, especially in Mexico and Spain, surveys have been carried out about the knowledge and position with regard to terms related to end-of life-decisions, ${ }^{2-5}$ where university students, caregivers and resident doctors, among other professionals involved in health care have been included, but there are very few surveys focused on the general population, which
Correspondence:

*Luz María Guadalupe Pichardo-García

E-mail: Ipichard@up.edu.mx
Date of reception: 13-06-2018

Date of acceptance: 05-01-2019

DOI: 10.24875/GMM.M19000247
Gac Med Mex. 2019;155:137-142

Contents available at PubMed www.gacetamedicademexico.com 
is ultimately the user and protagonist of those decisions, informed by the medical team. Another key aspect about the relevance of further investigating on the subject is that numerous investigations are referred to ill or terminally-ill population, ${ }^{6-8}$ but that which the individual not close to death thinks without a situation of pressure, has not been explored.

Although decision-making in extreme situations implies great challenges because "death, as a process, allows preparation in order for it to be consistent with personal convictions maintained throughout life, especially in those cases where its closeness is known", ${ }^{3}$ this type of decisions should be meditated on and understood prior to a crisis, during which numerous factors that limit the freedom of a person to make decisions about him/herself or his/her loved ones overlap. "Making correct moral decisions in the face of such circumstances can be difficult, especially for the emotional content extreme situations entail". ${ }^{10}$ Decisions made at the end of life imply an important and inescapable ethical burden related to the belief system of each person and constitute a part of individual moral.

It is important to know the understanding of end-oflife decisions-related terminology in apparently healthy general population, because it is in an adequate state of autonomy that allows reflection. From the perspective of researchers, it allows obtaining information without the bias of anguish and stress involved in extreme decisions and assessing the profile of the population of participating cities to further extend the sample size to even more cities and thus adequately focus educational and healthcare programs adjusted to the Mexican reality.

\section{Objective}

To determine the degree of knowledge of terms involved in end-of-life decisions the general population possesses in several cities of Mexico.

\section{Method}

Descriptive, exploratory, observational design, with surveys applied using the Survey Monkey program in Mexico City, Monterrey, Guadalajara and León, from October 2017 to January 2018. The questionnaire consists of 18 questions with nominal answers and demographic variables, which are divided into nominal (gender, city, religion, and occupation) and ordinal answers (level of education and health), as well as by interval (age).

The inclusion criteria were:

- Subjects older than 18 years.

- Individuals wanting to freely and voluntarily participate.

- People who did not work or had not worked in any profession within the health sector.

- Individuals not suffering from a terminal illness.

The questions include topics about the understanding and position of the general population with regard to different end-of-life terms. The survey was anonymous. Sampling was non-probabilistic. A pilot survey was conducted on 89 individuals to test the items. ${ }^{11}$ The final instrument was applied to 544 subjects. The collected data were analyzed by topics using the chisquare test. Statistical analysis was carried out with the R-Project program (version 3.3.3, The R Foundation for Statistical Computing Platform); in the first part, frequencies and percentages of demographic variables distribution were obtained, and in the second, an analysis with contingency tables was carried out to summarize the information contained in the surveys and measure the association between variables. Crossing of variables, which allows to determine the degree of association and to assess if they are statistically significant by means of the chi-square test and their adjustment with the likelihood ratio was carried out, in order to contrast the between-variable independence hypothesis; the between-variable independence hypothesis was rejected when the $p$-value was less than or equal to 0.05 . In addition, the contingency coefficient and Cramer values were calculated to assess the level of association when there was dependence between the answer and demographic variables. Finally, a proportionality test was carried out on the variables without homogeneity.

Due to the sample size, the study was regarded as a pilot test in the surveyed states.

\section{Results}

The survey was answered by 544 individuals: $70 \%$ women and $30 \%$ men; mean age was 35 years (range: 18 to 80 years). Distribution by age group was as follows: from 18 to 29 years, $25 \%$; from 30 to 39 years, $10 \%$; from 40 to 49 years, $21 \%$; from 50 to 59 years, $35 \%$; from 60 to 69 years, $8 \%$; and 70 years and older, $1 \%$; $83 \%$ indicated having good health, $16 \%$ regular and only $1 \%$, poor. By distribution according to the city, $58 \%$ resided in Mexico City, 
$10 \%$ in Guadalajara, $7 \%$ in Monterrey and $25 \%$ in León. As regards the level of education, college education accounted for $50 \%$, postgraduate for $23 \%$, high school or equivalent, $14 \%$, technical degree, 10 $\%$, and primary and secondary education for $3 \%$. Regarding occupation, $26 \%$ practiced a profession; $16 \%$ were homemakers; $16 \%$, students; $13 \%$ employees, and $7 \%$ were engaged in another activity; $5 \%$ were public servants, $5 \%$ were clerks, $4 \%$ were pensioned, $4 \%$ were traders, $2 \%$ were secretaries and $1 \%$ were domestic workers.

The answers by topic allowed perceiving which areas have to be worked on to clarify the terms. The frequencies are shown in Table 1.

Appendix 1 shows the significant demographic variables that influenced by topic $(p<0.05)$, the level of significance obtained by the contingency tables analysis to measure the degree of association each question and the demographic variables had is presented, with the variables with a positive relationship being indicated, which is part of this work's assessment of results.

As a summary, we point out the following relevant data:

1. People between 40 and 69 years of age, as well as professionals, knew well the palliative care term, which was indifferent for students and subjects younger than 29 years.

2. There was confusion in the population regarding the euthanasia and assisted suicide terms. For euthanasia, the most significant variables were age and religion. Surveyed subjects with postgraduate education adequately differentiated the term.

3. Regarding the term therapeutic obstinacy, the higher the level of education, the greater the disagreement with its application. People older than 50 years did not understand it.

4. Surveyed subjects with lower level of education, subjects between 18 and 39 years of age and students did not know the advance directive term or were indifferent about it. The rest of the population understood and accepted it (88\%).

5. Regarding the relevance of knowledge about end-of-life-related terms, $90 \%$ agreed that it facilitates decisions in critical situations. People younger than 30 years, as well as students, expressed indifference. The most associated variables were age, occupation, level of education and religion; $77 \%$ of surveyed subjects indicated that they did not receive useful information about these terms from health personnel or received it partially.

\section{Discussion}

This study explored the knowledge of the general population in Mexico City, Leon, Guadalajara and Monterrey, in surveyed subjects who considered themselves healthy (83\%), who failed to recognize and mixed up most end-of-life-related terms, and it is therefore urgent to cover this conceptual vacuum in order to promote informed decisions in the general population.

Regarding the comprehension of terms, the least known was therapeutic obstinacy (63\%) and the best known, palliative care (91\%). The term euthanasia is sufficiently known (75\%).

Indifference towards certain topics was explored: we found more indifference towards therapeutic obstinacy $(20 \%)$, which is consistent with lack of understanding. The results agree with Font Busquets et al. opinion, who point out that "it is necessary to find strategies that reduce this dissociation, including greater involvement of healthcare professionals in helping with advance planning of care and preparation of the advance directives document". ${ }^{2}$

Therapeutic obstinacy, defined in ethical terms, was rejected by the majority. These results are consistent with those of Ortiz Gonçalves et al., ${ }^{13}$ who point out that more than $50 \%$ of the population is against therapeutic obstinacy. In other topics, indifference ranged from 5 to $10 \%$ (Table 1), which was significant from the perspective of the comprehension of terms, since it should be nearly absent. It can be inferred that the topics were far from the interests of this type of population in the circumstances they were living in at the time of the survey.

The trend in favor of euthanasia was $50 \%$ and $40 \%$ against. Although much has been written on the subject, as pointed out by Morales et al., "on the other hand, there are hardly any studies on palliative care, medically assisted suicide and spiritual help".14 This trend is not exclusive to the general population, as it can be appreciated in the study by Toro Flores et al., ${ }^{15}$ who point out that $47 \%$ of pediatricians consider euthanasia as an acceptable practice.

It is important highlighting that when the topic of opportunely offered palliative care was addressed, the majority of the population agreed with this measure (91\%). For the type of answers about euthanasia and palliative care, it can be inferred that the population 
Table 1. Results of the pilot survey on knowledge about end-of-life-related terms ( $n=544)$

\begin{tabular}{|c|c|c|c|}
\hline & Agree (\%) & Indifferent (\%) & Disagree (\%) \\
\hline \multicolumn{4}{|l|}{ Topic 1. Palliative care } \\
\hline Q12. Do you know what palliative care is? & 71 & - & 29 \\
\hline $\begin{array}{l}\text { Q14. "Palliative care consists of adequate medical, psychological, family and spiritual } \\
\text { support in terminally ill patients, in order for death to be a less painful transition and } \\
\text { mourning to be more calm and peaceful". }\end{array}$ & 92 & 6 & 2 \\
\hline $\begin{array}{l}\text { Q19. Palliative care support in terminally ill patients can help in many cases to find meaning } \\
\text { to that last stage of life and live through it without anxiety and with more serenity. }\end{array}$ & 92 & 5 & 3 \\
\hline \multicolumn{4}{|l|}{ Topic 2. Euthanasia and assisted suicide } \\
\hline Q9. Do you know what assisted suicide is? & 76 & - & 24 \\
\hline Q10. Do you know what euthanasia is? & 96 & - & 4 \\
\hline Q11. Do you know if euthanasia and assisted suicide are the same? & 48 & - & 52 \\
\hline $\begin{array}{l}\text { Q13. What is your opinion about the following definition of euthanasia or assisted suicide: } \\
\text { "Any action or omission at the request of the patient that deliberately causes his/her } \\
\text { death in order for him/her not to experience pain or suffering"? }\end{array}$ & 74 & 7 & 19 \\
\hline Q20. A terminally ill patient may not be mentally lucid enough to ask for euthanasia. & 77 & 5 & 18 \\
\hline $\begin{array}{l}\text { Q21. I consider that the life of a person should be respected until its natural end. It would } \\
\text { never be admissible for the doctor to perform euthanasia, even if the patient asks for it. }\end{array}$ & 42 & 8 & 50 \\
\hline $\begin{array}{l}\text { Q22. In my opinion, it is preferable to die rather than to live without an adequate quality of } \\
\text { life. }\end{array}$ & 60 & 9 & 31 \\
\hline
\end{tabular}

Q15. Do you know what therapeutic obstinacy is?

Q16. Therapeutic obstinacy is to continue using treatments that no longer serve the terminally ill patient in any way.

\begin{tabular}{|c|c|c|}
\hline 38 & - & 62 \\
\hline 53 & 19 & 28 \\
\hline 48 & 5 & 47 \\
\hline 70 & 8 & 22 \\
\hline
\end{tabular}

Q17. The medical team must fight for the life of the patient until the last moment, even if there is no possibility of recovery.

Q18. It is unethical to obstinately continue applying measures that can no longer be useful in terminally ill patients.

\section{Topic 4. Advance directive}

Q23. Advance Directive is a legal document that is used for decision-making about treatments that are wanted or not to be applied, in case of losing consciousness and being in terminal phase.

Q24. The use of the Advance Directive document helps to avoid problems in decisions made in terminally ill patients.

\section{Topic 5. General knowledge about terms}

Q25. Knowing these terms will facilitate making calm and timely decisions in order to avoid and control the symptoms of those stages, such as pain, suffering, anxiety and depression, whether you were the patient or one of your close relatives?

\begin{tabular}{|l|c|c|c|}
\hline $\begin{array}{l}\text { Q26. The health staff usually informs completely and clearly what you need to know to } \\
\text { make the best decision for you or your patient in the last phase of life. }\end{array}$ & Yes (\%) & No (\%) & Sometimes (\%) \\
\hline
\end{tabular}

was divided regarding euthanasia, but the majority was in favor of opportunely offered palliative care. This is a very important point to be considered in public health policies, especially now that there is the obligation to have a palliative care department available in Mexico. Accessibility and quality of the palliative care will surely be decisive for the population to lean towards one or another end-of-life decision, 
where the information provided by health personnel has much to do.

Vermogeen et al. pertinently propose the following: ${ }^{16}$

In order for the quality of end-of-life care of family members to be improved, individual care professionals' communication skills need to be improved through changes in medical education and training programs (...) on the importance of promoting a healthcare culture where health professionals take into account the interests of the person suffering from an illness and those of his/her relatives, which is a common practice in palliative care. Future research is needed in order to identify interventions that can promote a holistic perspective within and between different medical care departments.

It can be concluded that the gender and level of education variables have an important influence on questions focused on concepts or definitions; unlike questions related to life and death, where the city and religion have a particular influence.

These issues not previously explored in Mexican surveys a have functional relevance. The higher the level of education, the greater the understanding of terminology, which indicates that there is a need to develop strategies for education on these terms in the poorly educated population. When reviewing the literature on the subject in general populations, only one article was found involving students, ${ }^{9}$ in 2007, aimed at advance directive and addressing other end-of-life decisions-related terms; the lack of knowledge was also clear.

The survey shows that only $30 \%$ of respondents felt that health personnel provide adequate information to choose the most appropriate treatments for terminally ill patients. This point, which is eminently ethical, implies a deficient doctor-patient relationship and especially fails to comply with the bioethical principles of beneficence and non-maleficence.

One of the limitations of the study was the disproportion of the sample in some states, which was compensated by a homogenization for the comparisons between demographic variables. The greatest strength was constituted by the stratification of results according to demographic variables, mainly age, religion, occupation and level of education, since these parameters had not been adequately studied by other researchers in Mexico.

\section{Conclusions}

The preparation for making an autonomous and weighted decision starts long before a critical situation arises. Therefore, it is urgent to design and support educational campaigns to promote a culture of good death in healthy people, who can reflect in advance on end-of-life terms, adapted to age, occupation, level of education, gender, religion, values, and culture, in different media. Subsequent surveys should investigate how, where, when and who would be responsible for this task, which would allow a more natural transition and without anguish in the inevitable moment of death, an essential part of living.

\section{Funding}

This research has been funded by Universidad Panamericana through Fomento a la Investigación UP 2017, code UP-Cl-2017-DER.

\section{References}

1. Cano-Valle F. ¿Es posible avanzar hacia una muerte digna?: la situación en México comparada con la situación en Holanda. Rev Inst Nal Enf Resp Mex. 2007;20:5-8.

2. Cucalón-Arenal JM, Marín-Ibáñez A, Cía-Gómez P, Blay-Cortés MG. Opinión de los universitarios sobre los cuidados al final de la vida. FEM. 2013;16:181-186.

3. Ibáñez-Masero O, Ortega-Galán AM, Carmona-Rega MI, Ruiz-Fernández MD, Cabrera-Troya J, García-Cisneros R, et al. El significado de morir dignamente desde la perspectiva de las personas cuidadoras: estudio fenomenológico. Enferm Clin. 2016;26:333-402.

4. Gaona-Flores VA, Campos-Navarro LA, Ocampo-Martínez J, Alcalá-Martínez E, Patiño-Pozas M. La "voluntad anticipada" y su conocimiento por médicos en hospitales de tercer nivel. Gac Med Mex. 2016;152:486-494.

5. Hildén HM, Louhiala P, Palo J. End of life decisions: attitudes of Finnish physicians. J Med Ethics. 2004:30:362-365

6. Sarmiento-Medina M, Vargas-Cruz S, Velásquez-Jiménez $C$, Sierra de Jaramillo M, Problemas y decisiones al final de la vida en pacientes con enfermedad en etapa terminal. Salud Publica. 2012;14:116-128.

7. Garduño-Espinosa A, Ham-Mancilla $O$, Méndez-Venegas J, Niembro ZAM, Pando CJM, Martínez BR, et al. Decisiones médicas al final de la vida. Recomendaciones para la atención a pacientes con enfermedades en estado terminal. Acta Pediatr Mex. 2006;27:307-316.

8. Tejedor-Torres JC, López de Heredia-Goya J, Herranz-Rubia N, Nicolás-Jiménez P, García-Muñoz F, Pérez-Rodríguez J, et al. Recomendaciones sobre toma de decisiones y cuidados al final de la vida en neonatología. An Pediatr (Barc). 2013;78:190.e1-190.e14.

9. Casas ML. Encuesta en población abierta sobre contenidos aplicables a los documentos de voluntades anticipadas (DVA). Rev Conamed. 2007:12:9-15.

10. Casas ML. Detección de necesidades familiares en la atención a pacientes en estado terminal y crónico en la Ciudad de México. Rev Conamed. 2006:11:19-24.

11. Pichardo-García LM, Casas-Martínez ML, Jaimes-Palomera M, Sotelo-Méndez AG, Sosa-Delgado AP, Quintero- Luna A, et al. Términos que facilitan las decisiones de fin de vida. ¿Es necesario prepararse para una buena muerte? An Med Hosp ABC. 2017;62:266-270.

12. Font-Busquets JM, De la Fuente Hidoine M, Lushchenkova O, Quintana S. Conocimiento y valoración por parte de los ciudadanos de Cataluña sobre las voluntades anticipadas y la atención al final de la vida. Medi Pa. 2014;21:153-159.

13. Ortiz-Gonçalves B, Santiago-Sáez A, Albarrán-Juan E, Labajo-González E, Perea-Pérez B. Elaboración de un cuestionario sobre conocimientos y actitudes de la población madrileña frente al final de la vida. Gaceta San. 2018;32:319-402.

14. Morales-Valdés G, Alvarado-Romero T, Zuleta-Castro R. Limitación del esfuerzo terapéutico en cuidados intensivos pediátricos: conocimiento y actitudes bioéticas del profesional médico. Rev Chil Pediatr. 2016;87: 116-200.

15. Toro-Flores R, López-González R, López-Muñoz JA. Conocimientos y actitudes de los pacientes críticos y sus familiares respecto a las directivas anticipadas y la toma de decisiones al final de la vida. Enferm Intensiva. 2017;28:21-30.

16. Vermorgeen M, De Vleminck A, Deliens L, Houttekier D, Spruytte N, Van-Audenhove $\mathrm{C}$, et al. Do physicians discuss end-of-life decisions with family members? A mortality follows back study. Patient Educ Couns. 2018;101:1378-1384. 
Gaceta Médica de México. 2019;155

Appendix 1. Significance values between demographic variables and each one of the questions ${ }^{*}$

Question

Q09. Do you know what assisted suicide is?

Q10. Do you know what euthanasia is?

Q11. Do you know if euthanasia and assisted suicide are the same?

Q12. Do you know what palliative care is?

Q13. What is your opinion about the following definition of euthanasia or assisted suicide: "any action or omission by request of the patient that deliberately causes his/ her death in order for him/her not to experience pain or suffering"?

Q14. "Palliative care consists of adequate medical, psychological, familiar and spiritual support in terminally ill patients in order for death to be a less painful transition and grieving to be more calm and peaceful"

Q15. Do you know what therapeutic obstinacy is?

Q16. Therapeutic obstinacy consists in continue using treatments that no longer serve the terminally ill patient in any sense.

Q17. The medical team must fight for the patient's life to the last moment, even if there are no possibilities of recovery.

Q18. It is unethical to obstinately continue applying measures that no longer can be useful in end-stage patients.

Q19. Palliative care support in terminally-ill patients can help, in many cases, to find sense to that last stage of life and live it without anguish and more serenity.

Q20. An end-stage patient can be in mental conditions that are not sufficiently lucid to request euthanasia.

Q21. I consider that the life of a person should be respected until its final end. It would never be admissible that the doctor applies euthanasia, even if the patient asks for it.

Q22. According to my criterion, it is preferable to die than to live without an adequate quality of life.

Q23. Advance Directive is a legal document that serves for decision-making about the treatments the patient wishes to have applied and not in case of losing consciousness and being at end-stage phase.

Q24. The use of the Advance Directive document helps avoiding problems in decisions made in terminally ill patients.

Q25. If you know these terms would it be easier to make serene and timely decisions in order to avoid and control symptoms of that stage, such as pain, suffering, anxiety and depression, whether you or one of your close relatives were the patient?

Q26. Health personnel usually informs you thoroughly and clearly on what you need to know to make the best decision for you or for your patient in the last phase of life?

\section{City Education Health Gender Religion Age Occupation Total}

$(p<0.05)$. The variables with the highest association were level of education, age, occupation and religion, as well as the questions most related to these parameters. 